\title{
Decitabine-containing G-CSF priming regimen overcomes resistance of primary mediastinal neoplasm from early T-cell precursors to conventional chemotherapy: a case report
}

This article was published in the following Dove Press journal: OncoTargets and Therapy

\author{
Yuhuan Yang ${ }^{1, *}$ \\ Shuna Yaol,* \\ Jiuyang Zhang' \\ Zheng Yan' \\ Junfeng Chu' \\ Haiying Wang' \\ Zhihua Yao' \\ Fan Zhang ${ }^{2}$ \\ Qingxin $\mathrm{Xia}^{3}$ \\ Yanyan Liu'
}

'Department of Internal Medicine, Affiliated Cancer Hospital of Zhengzhou University, Zhengzhou, Henan, People's Republic of China; ${ }^{2}$ Department of Central Laboratory, Affiliated Cancer Hospital of Zhengzhou University, Zhengzhou, Henan, People's Republic of China; ${ }^{3}$ Department of Pathology, Affiliated Cancer Hospital of Zhengzhou University, Zhengzhou, Henan, People's Republic of China

*These authors contributed equally to this work

Correspondence: Yanyan Liu Department of Internal Medicine, Affiliated Cancer Hospital of Zhengzhou University, 127 Dong Ming Road,

Zhengzhou, Henan 450008, People's

Republic of China

Email yyliu@zzu.edu.cn

Qingxin Xia

Department of Pathology, Affiliated Cancer Hospital of Zhengzhou University, I 27 Dong Ming Road, Zhengzhou, Henan 450008, People's Republic of China Email13838173710@I39.com

\begin{abstract}
Early T-cell precursor (ETP) leukemia represents a new subtype of T-lymphoblastic leukemia/lymphoma with unique immunophenotypes expressing T-cell and one or more of the myeloid/stem cell markers. Here, we report a young patient who had primary mediastinal mass and pleural effusion without bone marrow involvement. A CT-guided mediastinal biopsy and flow cytometry analysis of the pleural effusion revealed the blast cells to have complicated immunophenotypes: strongly expressed T-cell antigen CD7, myeloid-lineage antigens CD33 and CD13 and stem cell markers cTdT, CD34, and HLADR; dimly expressed myeloid-lineage specific antigen cMPO and B-cell antigen cCD79a; but did not express T-cell specific antigen cytoplasmic CD3 and B-cell specific antigen CD19. Clonal T-cell receptor rearrangement eventually determined the cell of origin from ETPs, not myeloblasts. The patient showed primary resistance to lymphoid and myeloiddirected induction therapy. Finally, low-dose decitabine combined with modified-CAG regimen induced a complete remission and allogeneic stem cell transplantation was performed as consolidation. The case indicates a primary mediastinal neoplasm from ETP with distinctive immunophenotype from leukemia type. Low-dose decitabine and modified-CAG regimen in combination with allogeneic stem cell transplantation may improve the outcome of patient. Keywords: early T-cell precursor, decitabine, G-CSF priming, T-lymphoblastic lymphoma
\end{abstract}

\section{Introduction}

T-lymphoblastic leukemia/lymphoma (T-ALL/LBL) is a precursor lymphoid neoplasm that occurs in bone marrow and blood (T-ALL) or involves the thymus, lymph nodes, or extranodal sites (T-LBL). The thymus is the most common site involved by T-LBL, which is located in the anterior mediastinum and holds the key to T-cell development. There are four stages of intrathymic differentiation related to T-ALL/LBL according to the antigens expressed: 1) pro-T/T-I, 2) pre-T/T-II, 3) cortical T/T-III, and 4) medullary T/T-IV. ${ }^{1}$ Early T-cell precursors (ETPs) usually belong to pro-T and pre-T cells and retain multiple potentials for T-cells and natural killer cells as well as myeloid lineage and dendritic cell differentiation. ${ }^{2,3}$ ETP-ALL, as a neoplasm from ETP, has been defined to be a new subtype of T-ALL/LBL in 2016 WHO classification. ${ }^{4}$ The diagnosis of ETP-ALL is mainly based on a set of unique immunophenotypes: expresses CD7, cytoplasmic CD3 (cCD3), and one or more of the myeloid/stem cell markers CD34, CD117, 
HLA-DR, CD33, CD13, CD11b, and CD65, but lacks CD8, CD1a, and MPO. ${ }^{5}$ The mutation profiling of ETP-ALL is more similar to that of myeloid leukemia than to those of other T-cell leukemias. ${ }^{4}$ Recent studies have shown that patients with ETP-ALL have a similar clinical outcome as those with other T-ALL phenotypes, although initial studies suggested a very poor prognosis. ${ }^{6}$

Here we reported a patient having a clear primary lesion in the mediastinum and pleural effusion without bone marrow involvement. He possessed detectable multi-lineage antigens of stem cells, T-cells, B-cells, myeloid cells, and dendritic cells. Although there was a similar immunophenotypic feature to myeloid sarcoma with dim expression of cMPO and without cCD3, he was diagnosed as ETP-LBL depending on clonal T-cell receptor (TCR) rearrangement. The case presented with primary resistance to lymphoid and myeloiddirected induction therapy. Fortunately, novel myeloid-directed salvage therapy, decitabine-containing G-CSF priming regimen, induced a complete remission and allogeneic stem cell transplantation further improved the outcome.

\section{Case presentation}

A 22-year-old male was admitted to our hospital for chest pain and dyspnea in November 2017. He has gotten a continuous pain in anterior chest since July 2017. After comprehensive CT scan was done at his local hospital, a large mediastinal mass was found. A CT-guided mediastinal biopsy in combination with immunohistochemistry showed that T-cell, stem cell, and myeloid-lineage antigens were present in the neoplasm tissues: $\mathrm{LCA}+, \mathrm{TdT}+$, $\mathrm{CD} 7+, \mathrm{CD} 34+, \mathrm{Ki}-67$ (80\%), $\mathrm{MPO}+, \mathrm{CD} 33+, \mathrm{CD} 8-$, CD1a-, CD117-, CD15-, cCD3-, CD2-, CD10-, CD19-, PAX5-, CK-, S-100- (Figure 1). Since bone marrow was not involved by the analysis of aspiration and biopsy with routine staining and flow cytometry, he was diagnosed as T-LBL with myeloid differentiation. CHOP (cyclophosphamide, vincristine, epirubicin, and prednisone) and DICE (dexamethasone, ifosfamide, etoposide, and cisplatin) regimens were given at the local hospital, but the neoplasm continued to deteriorate.

Multiple supraclavicular lymph node swellings were palpated with a maximal diameter of about $2 \mathrm{~cm}$ at our hospital. The patient had weak respiratory sounds and large amounts of hydrothorax in left chest cavity were observed by ultrasound. After the pleural effusion was drained, PET-CT scan was performed, which revealed a bulky mass in anterior superior mediastinum (SUVmax 8.1) and many enlarged lymph nodes in bilateral cervical,

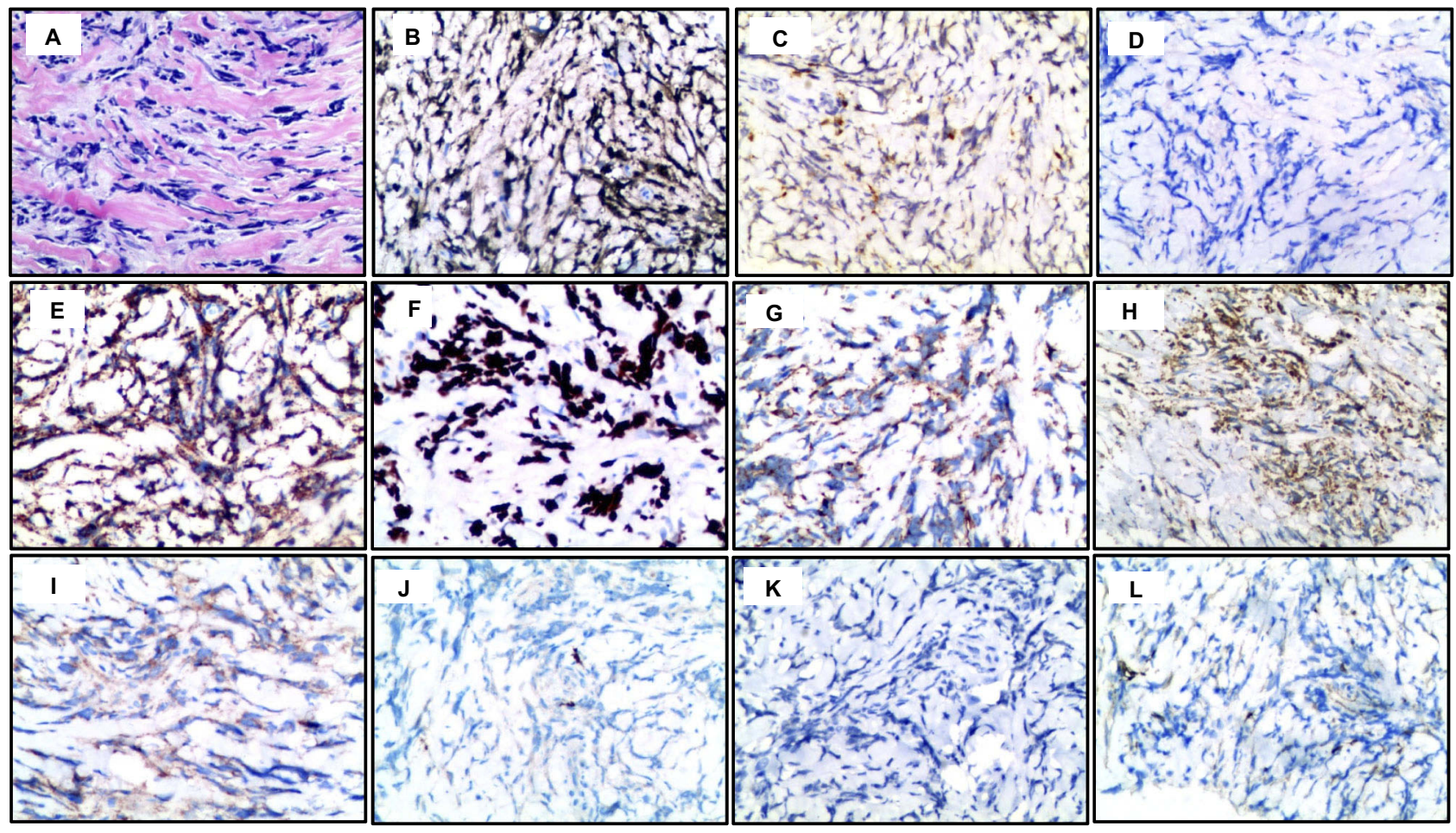

Figure I The immunohistochemical staining of the mediastinum mass (magnification, $\times 200)$. (A) H\&E staining. The neoplasm expressed TdT (B), CD7 (C), MPO (E), Ki67 $(80 \%)(\mathbf{F})$, CD34 (G), CD33 (H), and LCA (I), while it did not express CCD3 (D), CD8 (J), PAX5 (K), and CDIa (L). 
supraclavicular, subclavian, hilar, axillary, and retroperitoneal regions (SUVmax from 3.0 to 4.6) (Figure 2). Bone marrow was also negative by the examination of aspiration and biopsy. Comprehensive blood tests revealed elevated lactate dehydrogenase and erythrocyte sedimentation rate. The immunohistochemistry slides were assessed by our pathologists. Flow cytometry analysis of blast cells in the pleural effusion found a single population expressing multi-lineage markers (Figure 3). Bright expression of T-cell antigen CD7 and dim expression of B-cell antigen cCD79a were seen, though T-cell specific antigen cCD3 and B-cell specific antigen CD19 were negative. Myeloidlineage specific antigen cMPO was dimly expressed while myeloid-lineage antigens CD33 and CD13 were brightly
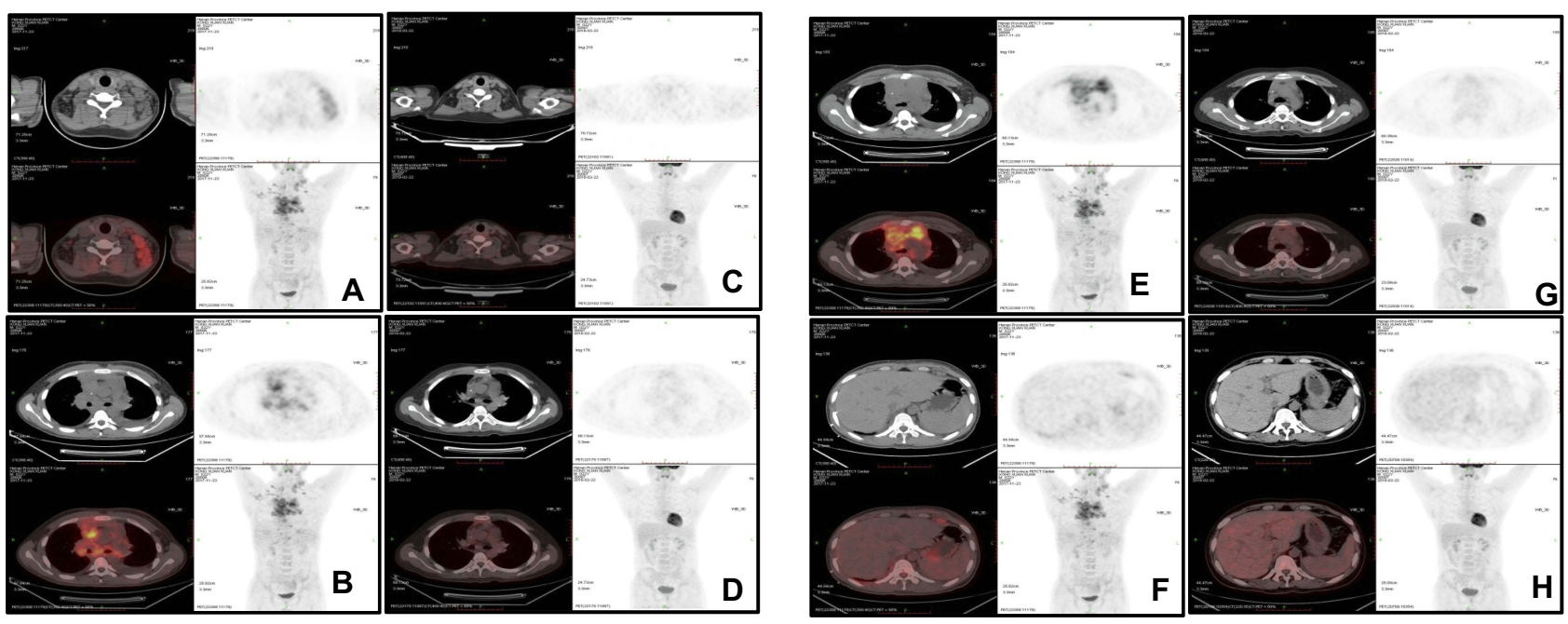

Figure 2 The PET-CT imaging of the lesions before and after treatment. In comparison with before treatment (A, B, E, F), the masses disappeared in left cervix, mediastinum, and peritoneal cavity after the completion of therapy $(\mathbf{C}, \mathbf{D}, \mathbf{G}, \mathbf{H})$.
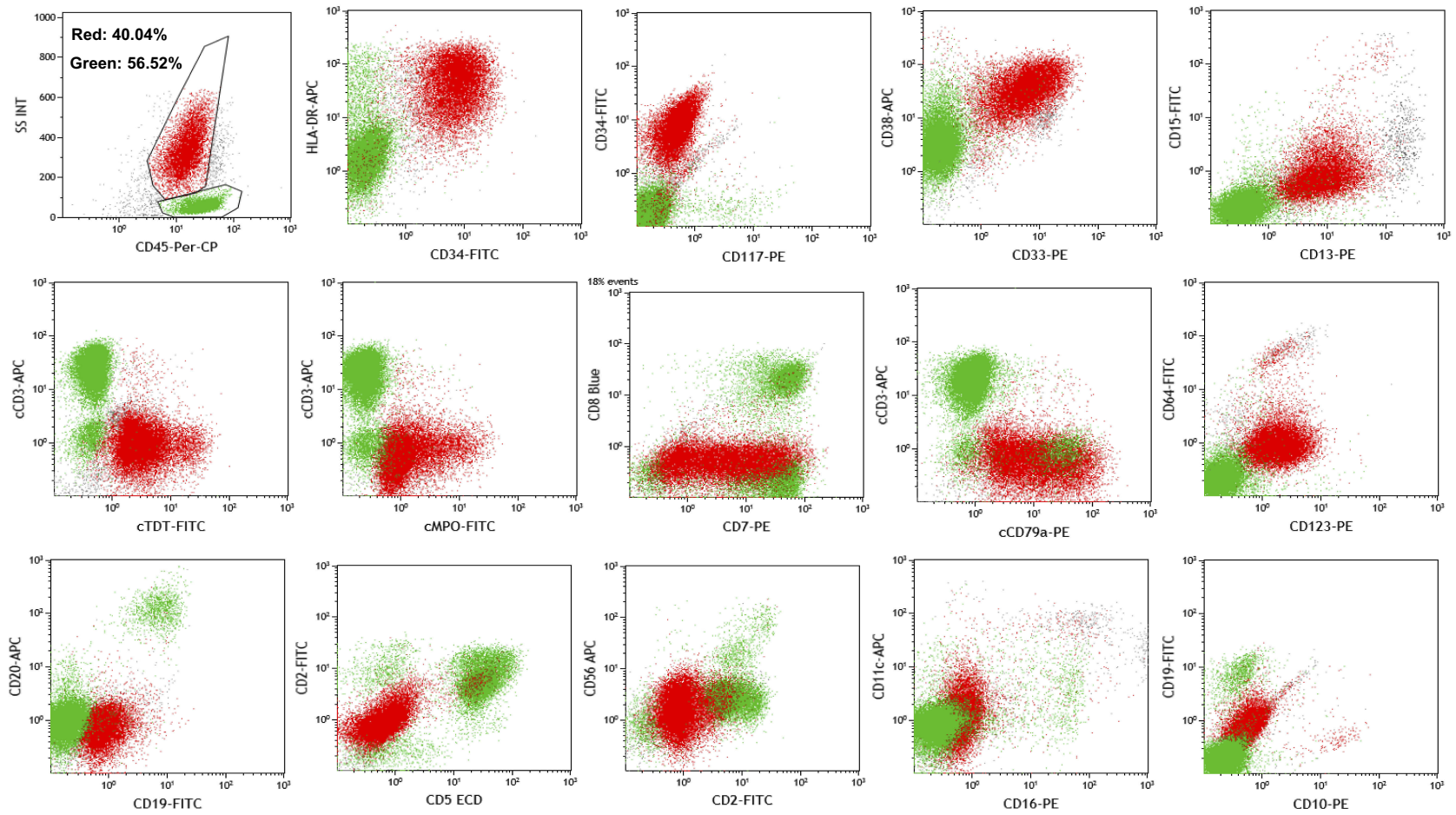

Figure 3 Flow cytometry analysis of lineage antigens of blast cells in pleural effusion. The blast cells brightly expressed CD7, CD33, CDI3, cTdT, CD34, HLA-DR, and CD38, dimly expressed cCD79a, cMPO, and CDI23, but did not express CCD3, CDI9, CD20, CDII7, CD2, CD5, CD8, CDI0, CDI5, CDI6, and CD56. 
expressed. We also found bright expression of stem cell markers cTdT, CD34, HLA-DR, and CD38, and dim expression of blastic plasmacytoid dendritic cell marker CD123. Other specific markers were negative, including stem cell marker CD117, monocyte markers CD15 and CD11c, T-cell markers CD1a, CD2, CD4 and CD8, NK cell markers CD16 and CD56, and B-cell markers CD10, $\mathrm{CD} 19$, and CD22. At first sight, the neoplasm met the criteria for myeloid sarcoma due to having cMPO expression, but not cCD3. However, clonal TCR gene rearrangement was found, which suggested an origin of T-cell precursor (Figure 4). ETP neoplasm was postulated to originate from a subset of cells that immigrated from the bone marrow into the thymus but were not yet irreversibly committed to the T-cell lineage and retained the potential for myeloid/dendritic cell differentiation. In contrast to definitive ETP-ALL, the patient did not have the bone marrow involvement. Some markers were consistent with ETP-ALL phenotype, including positive CD7, CD34, HLA-DR, CD33, and CD13, and negative CD1a and CD8, whereas others were not consistent, such as positive cMPO and negative cCD3. Eventually, the case was considered to be ETP-LBL with some distinctive features from ETP-ALL.
A 4-week hybrid regimen of myeloid and lymphoiddirected therapy, DA (daunorubicin and cytarabine) plus VLP (vindesine, pegaspargase, and prednisone), was given at our hospital. Nevertheless, the masses did not shrink significantly after the completion of the 4-week schedule. So far, the optimal treatment for patients with a neoplasm from ETP remains elusive, especially for those with primary resistance to conventional chemotherapy. Since the case was positive for myeloid-lineage antigens, myeloiddirected salvage regimens might be a suitable choice. CAG regimen (cytarabine $10 \mathrm{mg} / \mathrm{m} 2 \mathrm{q} 12 \mathrm{~h}$ for $10-14$ days and aclarubicin $20 \mathrm{mg} / \mathrm{d}$ for 4 days with G-CSF priming) had been reported to produce responses in refractory patients with acute myeloid leukemia. ${ }^{7} \mathrm{CAG}$ regimen in combination with decitabine, a DNA methyltransferase inhibitor approved to treat patients with myelodysplasia syndrome, had also been reported to improve CR rate in patients with newly diagnosed MDS-EB and AML-MRC. ${ }^{8}$ However, the optimal dosage and schedule of decitabine were rather challenging when used in combination with other drugs. Due to the patient's poor performance status, low-dose decitabine $(10 \mathrm{mg} / \mathrm{d}$ subcutaneous injection, twice a week for three times) combined with modifiedCAG regimen (cytarabine of $10 \mathrm{mg} / \mathrm{m} 2 \mathrm{q} 12 \mathrm{~h}$ for 10 days
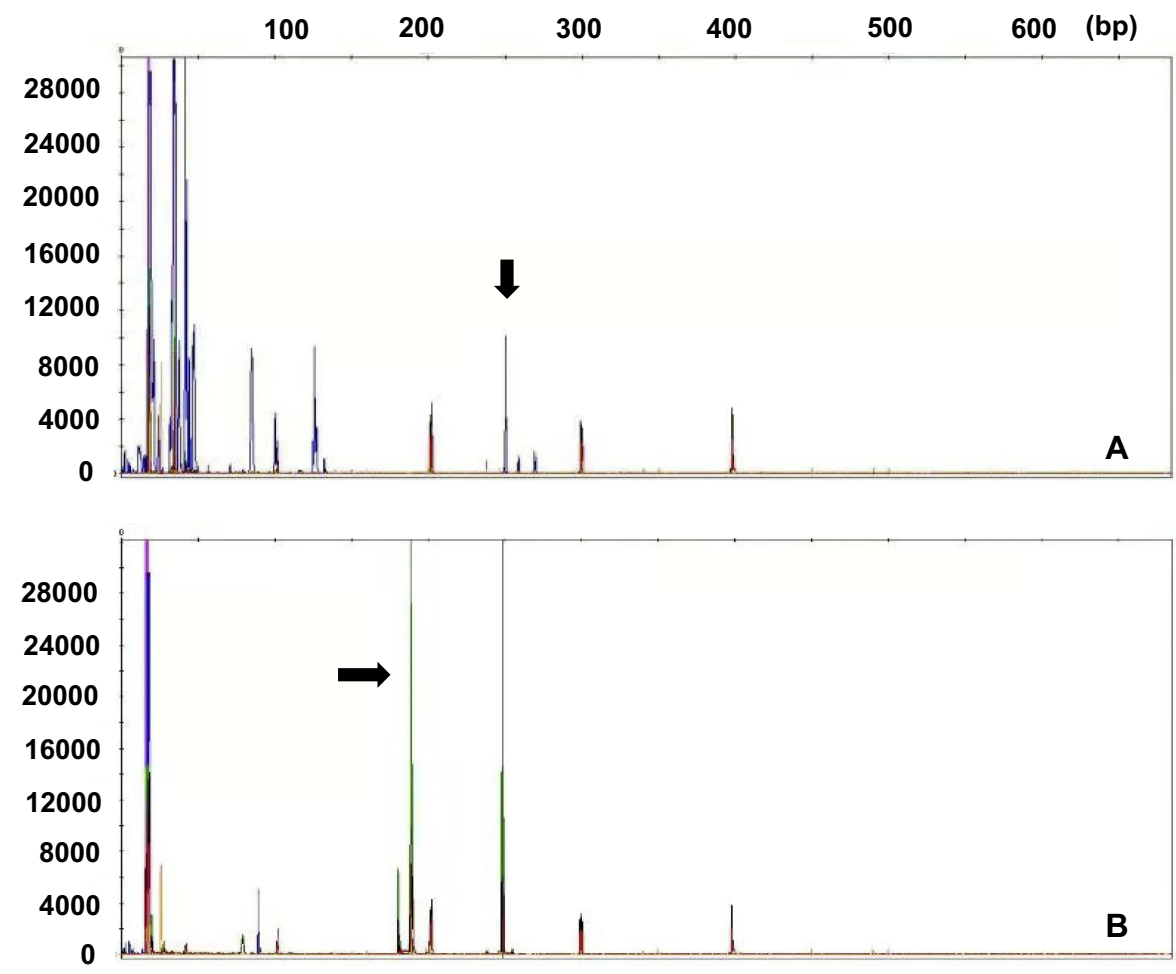

Figure 4 Conal T-cell receptor (TCR) rearrangement of blast cells. Clonal gene rearrangement was observed in TCR beta VJII (A) and TCR delta VD/DD/DJ (B) fragments. In panel (A), the arrow indicates the monoclonal peak of TCR beta VJII. In Panel (B), the arrow indicates the monoclonal peak of TCR delta VD/DD/DJ. 
and aclarubicin of $20 \mathrm{mg} / \mathrm{d}$ for 4 days with pegylated $6 \mathrm{mg}$ G-CSF priming) was given. Encouragingly, superficial masses disappeared following a treatment cycle. After two cycles of treatment, the patient achieved a $\mathrm{CR}$ as evaluated by PET/CT scan (Figure 2). Generally, adverse effects were tolerable and reversible, including grade IV neutropenia, anemia, thrombocytopenia, and grade I transaminase lift. Four cycles of high-dose methotrexate-based regimens were then taken as the prophylaxis of the central nervous system and intensification therapy and then additional two cycles of low-dose decitabine plus modified-CAG regimen were administered as re-induction and consolidation therapy. Since HLA-identical donor was not found, the patient's father was selected for mismatchedallogeneic stem cell transplantation. A successful operation was performed in June 2018. By the end of June 2019, the patient had been maintaining a CR status.

\section{Discussion and conclusion}

The classical model of hematopoiesis postulates that pluripotent hematopoietic stem cells of bone marrow differentiate into common myeloid-erythroid progenitors and common lymphoid progenitors. Some lymphoid progenitors migrate to the thymus for T-cell development. However, further studies have found that the myeloid-lineage potential persists even as the lineage branches segregate toward $\mathrm{T}$ - or B-cells. ${ }^{2,3}$ ETP-ALL has been considered as a neoplasm originated from ETP, indicating a limited early T-cell differentiation with frequent expression of the markers of stem cells and myeloid cells. ${ }^{4}$ The case represents an alternative neoplasm from ETP, referred to as ETP-LBL, with different immunophenotypic features from ETP-ALL. Owing to expressing cMPO, but not cCD3, the neoplasm should be differentiated with myeloid sarcoma. For myeloid sarcoma, the expression of CD7 is usually weak, but cMPO is often strong in granulocytic sarcoma. The dim expression of cMPO weakens the significance of diagnosing myeloid sarcoma. Anti-MPO antibodies have been shown to react with B-lymphoblastic leukemia/lymphoma on flow cytometry or immunohistochemistry as a non-specific staining. ${ }^{9,10}$ In fact, the dim expression of B-cell antigen cCD79a was seen in the case. Mediastinal myeloid sarcoma is a rare disease. According to the definition of 2015 WHO classification of thymus tumors, mediastinal myeloid sarcoma should be considered to exclude if lymphoid-antigens are coexpressed with myeloid-lineage antigens. Eventually, clonal TCR gene rearrangement confirmed the diagnosis of ETP neoplasm.
T-LBL originates from a thymic lymphocytes and presents with a primary mass in the anterior mediastinum, which often grows rapidly and sometimes leads to respiratory failure. T-LBL usually has a better prognosis than TALL, however, its prognostic factors remain ambiguous compared to T-ALL. ${ }^{11}$ Previous studies did not show its association of T-LBL with clinical features, such as age, LDH level, and extranodal lesion, except the involvement of the central nervous system. ${ }^{12}$ One or both of the myeloidassociated antigens $\mathrm{CD} 13$ and $\mathrm{CD} 33$ are expressed in $19-32 \%$ of the patients with T-LBL. ${ }^{13,14}$ Till now, studies on the prognostic significance of myeloid-lineage markers are limited. Our case indicates a poorer outcome of ETP-LBL in the setting of conventional therapy. As a novel therapy for myeloid neoplasms, CAG regimen alone or with decitabine has achieved an obvious response in patients with refractory acute lymphoblastic leukemia. ${ }^{15,16}$ However, the predominant subtype who benefited from it is unclear. The successful treatment of the present case with low-dose decitabine in combination with modified-CAG regimen indicates the significance of this novel regimen for treating the ETP neoplasms. Finally, allogeneic stem cell transplantation eventually extended the survival time of the patient.

In conclusion, comprehensive lineage antigens should be examined to make the diagnosis of neoplasms from ETP due to their complicated immunophenotypes. Sometimes, the examination of antigen receptor genes plays a critical role in differential diagnosis. We speculate that ETP neoplasm should be a heterogeneous disease in clinical characteristics, immunophenotypic features, and prognosis. Novel myeloid-directed novel therapy with allogeneic stem cell transplantation may improve the outcome in patients with primary resistance to conventional therapy.

The patient has provided written informed consent for publication. This case report does not require institutional approval to publish the case details.

\section{Acknowledgments}

The authors thank the patient and his families and all of physicians and nurses who took care of the patient. This report was supported by the National Natural Science Foundation of China (No. 81071938) and the Science and Technology Projects of Henan Province Education Department (No. 81470365).

\section{Disclosure}

The authors report no conflicts of interest in this work. 


\section{References}

1. Bene MC, Castoldi G, Knapp W, et al. Proposals for the immunological classification of acute leukemias. European Group for the Immunological Characterization of Leukemias (EGIL). Leukemia. 1995;9(10):1783-1786.

2. Bell JJ, Bhandoola A. The earliest thymic progenitors for $\mathrm{T}$ cells possess myeloid lineage potential. Nature. 2008;452(7188):764-767. doi: 10.1038 /nature 06840

3. Luc S, Luis TC, Boukarabila $\mathrm{H}$, et al. The earliest thymic $\mathrm{T}$ cell progenitors sustain B cell and myeloid lineage potential. Nat Immunol. 2012;13(4):412-419. doi:10.1038/ni.2255

4. Arber DA, Orazi A, Hasserjian R, et al. The 2016 revision to the World Health Organization classification of myeloid neoplasms and acute leukemia. Blood. 2016;127(20):2391-2405. doi:10.1182/blood2016-03-643544

5. Coustan-Smith E, Mullighan CG, Onciu M, et al. Early T-cell precursor leukaemia: a subtype of very high-risk acute lymphoblastic leukaemia. Lancet Oncol. 2009;10(2):147-156. doi:10.1016/S14702045(08)70314-0

6. Jain N, Lamb AV, O'Brien S, et al. Early T-cell precursor acute lymphoblastic leukemia/lymphoma (ETP-ALL/LBL) in adolescents and adults: a high-risk subtype. Blood. 2016;127(15):1863-1869. doi:10.1182/blood-2015-08-661702

7. Xu J, Lv TT, Zhou XF, Huang Y, Liu DD, Yuan GL. Efficacy of common salvage chemotherapy regimens in patients with refractory or relapsed acute myeloid leukemia: a retrospective cohort study. Medicine (Baltimore). 2018;97(39):e12102. doi:10.1097/MD.0000000000012102

8. Liu J, Jia JS, Gong LZ, et al. [Efficacy and safety of decitabine in combination with G-CSF, low-dose cytarabine and aclarubicin in MDS-EB and AML-MRC]. Zhonghua Xue Ye Xue Za Zhi. 2018;39 (9):734-738. doi:10.3760/cma.j.issn.0253-2727.2018.09.006
9. Leong CF, Kalaichelvi AV, Cheong SK, Hamidah NH, Rahman J, Sivagengei K. Comparison of myeloperoxidase detection by flow cytometry using two different clones of monoclonal antibodies. Malays J Pathol 2004;26(2):111-116.

10. Nakase K, Sartor M, Bradstock K. Detection of myeloperoxidase by flow cytometry in acute leukemia. Cytometry. 2015;34(4):198-202.

11. Marks DI, Rowntree C. Management of adults with T-cell lymphoblastic leukemia. Blood. 2017;129(9):1134-1142. doi:10.1182/blood2016-07-692608

12. Goldberg JM, Silverman LB, Levy DE, et al. Childhood T-cell acute lymphoblastic leukemia: the Dana-Farber Cancer Institute acute lymphoblastic leukemia consortium experience. J Clin Oncol. 2003;21 (19):3616-3622. doi:10.1200/JCO.2003.10.116

13. Uckun FM, Sather HN, Gaynon PS, et al. Clinical features and treatment outcome of children with myeloid antigen positive acute lymphoblastic leukemia: a report from the Children's Cancer Group. Blood. 1997;90(1):28-35.

14. Khalidi HS, Chang KL, Medeiros LJ, et al. Acute lymphoblastic leukemia. Survey of immunophenotype, French-American-British classification, frequency of myeloid antigen expression, and karyotypic abnormalities in 210 pediatric and adult cases. Am J Clin Pathol. 1999;111(4):467-476. doi:10.1093/ajcp/111.4.467

15. Zhou K, Song Y, Zhang Y, et al. Efficacy and safety of G-CSF, lowdose cytarabine and aclarubicin in combination with 1-asparaginase, prednisone in the treatment of refractory or relapsed acute lymphoblastic leukemia. Leuk Res. 2017;62:29-33. doi:10.1016/j. leukres.2017.09.016

16. Qin T, Youssef EM, Jelinek J, et al. Effect of cytarabine and decitabine in combination in human leukemic cell lines. Clin Cancer Res. 2007;13(14):4225-4232. doi:10.1158/1078-0432.CCR06-2762
OncoTargets and Therapy

\section{Publish your work in this journal}

OncoTargets and Therapy is an international, peer-reviewed, open access journal focusing on the pathological basis of all cancers, potential targets for therapy and treatment protocols employed to improve the management of cancer patients. The journal also focuses on the impact of management programs and new therapeutic

\section{Dovepress}

agents and protocols on patient perspectives such as quality of life, adherence and satisfaction. The manuscript management system is completely online and includes a very quick and fair peer-review system, which is all easy to use. Visit http://www.dovepress.com/ testimonials.php to read real quotes from published authors. 